ARTICLE

Received 3 Feb 2014 | Accepted 6 Jun 2014 | Published 11 Jul 2014 DOl: 10.1038/ncomms5360

\title{
Mechanical gate control for atom-by-atom cluster assembly with scanning probe microscopy
}

Yoshiaki Sugimoto ${ }^{1}$, Ayhan Yurtsever ${ }^{2}$, Naoki Hirayama1, Masayuki Abe ${ }^{3, \dagger} \&$ Seizo Morita ${ }^{2}$

Nanoclusters supported on substrates are of great importance in physics and chemistry as well as in technical applications, such as single-electron transistors and nanocatalysts. The properties of nanoclusters differ significantly from those of either the constituent atoms or the bulk solid, and are highly sensitive to size and chemical composition. Here we propose a novel atom gating technique to assemble various atom clusters composed of a defined number of atoms at room temperature. The present gating operation is based on the transfer of single diffusing atoms among nanospaces governed by gates, which can be opened in response to the chemical interaction force with a scanning probe microscope tip. This method provides an alternative way to create pre-designed atom clusters with different chemical compositions and to evaluate their chemical stabilities, thus enabling investigation into the influence that a single dopant atom incorporated into the host clusters has on a given cluster stability.

\footnotetext{
${ }^{1}$ Department of Electrical, Electronic and Information Engineering, Graduate School of Engineering, Osaka University, 2-1, Yamada-Oka, Suita, Osaka 5650871, Japan. ${ }^{2}$ Nanoscience and Nanotechnology Center, The Institute of Scientific and Industrial Research, Osaka University, 8-1 Mihogaoka, Ibaraki, Osaka 567-0047, Japan. ${ }^{3}$ Department of Materials Science and Engineering, Graduate School of Engineering, Nagoya University, Furo-cho, Chikusa-ku, Nagoya 464-8603, Japan. †Present address: Center for Science and Technology under Extreme Conditions, Graduate School of Engineering Science, Osaka University, 1-3, Machikaneyama-Cho, Toyonaka, Osaka 560-8531, Japan. Correspondence and requests for materials should be addressed to Y.S. (email: sugimoto@afm.eei.eng.osaka-u.ac.jp).
} 
$\mathrm{N}$ anoclusters supported on substrates are of great importance in physics and chemistry as well as in technical applications, such as single-electron transistors ${ }^{1,2}$, nanocatalysts $^{3-5}$ and quantum computing devices ${ }^{6}$. The properties of nanoclusters are highly sensitive to size and chemical composition. Despite their inert bulk properties, for example, nanosized Au clusters exhibit high catalytic activities for a wide variety of chemical reactions ${ }^{3}$, showing clear quantum size effects on catalytic behaviour ${ }^{5}$. The development of methods to fabricate atomically well-defined clusters is therefore necessary for a fundamental understanding of their properties and for designing better materials and devices.

Various approaches have been developed to create nanoclusters with different functionalities on surfaces. Among these methods, the self-assembling is the most widely used method for fabricating nanoclusters, where atoms/molecules are evaporated onto a surface followed by thermal annealing, leading to the spontaneous formation of clusters ${ }^{7}$. To unveil the unique properties of these clusters in practical applications, it is essential to control their size and uniformity. Although the uniformity of cluster arrays has been achieved using the so-called template-induced cluster formation method ${ }^{8,9}$, these methods do not provide direct access to the precise determination of the number of atoms involved in a cluster structure once they are deposited on surfaces.

Complementary to these methods, the scanning probe method offers precise positioning capabilities with atomic precision ${ }^{10}$. These capabilities have led to the creation of various artificial nanostructures atom-by-atom, mainly at cryogenic temperatures ${ }^{11-14}$. This method was later extended to room temperature (RT) by means of atomic force microscopy (AFM) ${ }^{15}$, which provides an opportunity to directly measure the forces that induce the atomic motion in the manipulation process ${ }^{16-18}$. Despite these achievements, assembly of the well-defined clusters exhibiting high thermal stability at RT and the precise determination of the cluster size and hence of the number of atoms involved in a cluster structure remains a challenging task ${ }^{19}$. More importantly, assembling of atomically well-defined clusters from multi-elements at RT has not been demonstrated yet despite their wide range of technical applications. The investigation of the structure and the stability of these bimetallic clusters can contribute to our understanding of the interaction of the doped atom with the metal clusters.

In this study, we propose an alternative approach to the assembly of various nanoclusters atom-by-atom using the tip of a scanning probe microscope (SPM) at RT. This method is based on the transfer of single diffusing atoms among nanospaces (NSs) governed by gates, which can be opened in response to the chemical interaction force with the SPM tip. This method provides a way to create pre-designed atom clusters with different chemical compositions and to evaluate their chemical stabilities.

\section{Results}

Transfer of diffusing adsorbates by atom gating. Half-unit cells (HUCs) of the $\mathrm{Si}(111)-(7 \times 7)$ surface serve as NS arrays to confine individual adsorbates diffusing on the surface. Single atoms deposited on the surface, such as group IV elements ( $\mathrm{Si}, \mathrm{Sn}$ and $\mathrm{Pb}$ ) and noble metals (Ag and $\mathrm{Au}$ ), thermally diffuse within the NSs at RT as demonstrated in Fig. 1. A potential energy barrier existing between NSs prohibits inter-NS thermal diffusion. The diffusion rate within the NS is so large that a SPM cannot resolve the specific adsorption sites offered by the surface at $\mathrm{RT}^{20}$ (further information can be found in Supplementary Note 1). The different adsorbate atoms on the surface appear differently in the SPM images owing to differences in their diffusion properties (Fig. 1b-i).

The clusters with predetermined compositions, such as $\mathrm{Au}_{12}$, $\mathrm{Ag}_{12}$ and $\mathrm{Au}_{5} \mathrm{~Pb}$, can be formed by collecting single atoms from the surrounding NSs into a predefined NS with successive gate controls. Hereafter, $\mathrm{Au}_{N}$ represents the $\mathrm{Au}$ cluster with $\mathrm{N} \mathrm{Au}$ atoms and the term 'cluster' refers to an ensemble of foreign atoms bonded to the $\mathrm{Si}(111)-(7 \times 7)$ surface. An adsorbate diffusing within a NS can be transferred to an adjacent NS by the tip proximity near the border, but slightly shifted away from the NS where the diffusing atom is located. The gate associated with the potential energy barrier that hinders the mobility of an adsorbate between NSs can be opened by the following consecutive steps. First, the tip is positioned over the predefined site marked with a cross as in Fig. 1a, and then the tip-to-surface
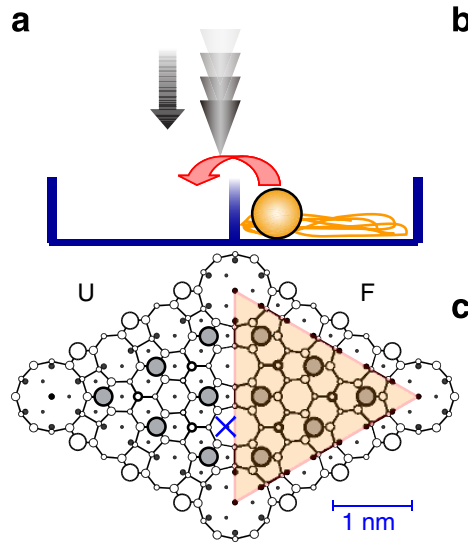

b
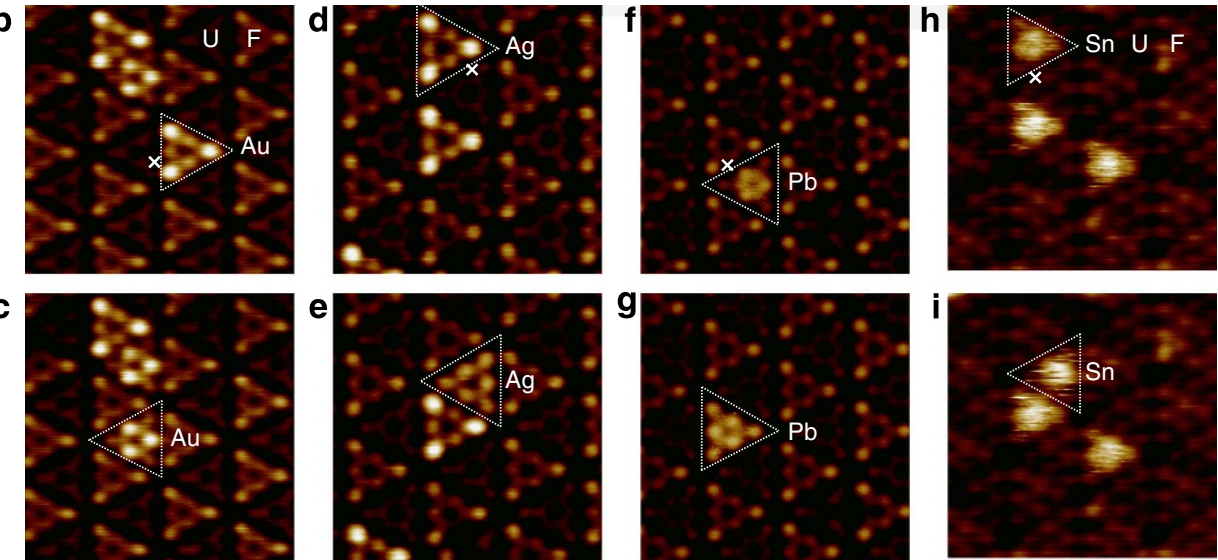

Figure 1 | Demonstrations of the transfer of a single atom by gate control. (a) A HUC in the $\mathrm{Si}(111)-(7 \times 7)$ surface works as a NS of an equilateral triangle with side lengths of $2.7 \mathrm{~nm}$. F and $\mathrm{U}$ denote faulted and unfaulted HUC, respectively. The adsorbate atom travels among the various potential minima in a NS; these minima are known to be multi-coordinate sites rather than the tops of Si adatoms and rest atom sites ${ }^{26}$. The tip gradually approaches the position as indicated by a cross in the $\mathrm{Si}(111)-(7 \times 7)$ unit cell for the gate-opening process. This process initiates the atom transfer between two NSs. $(\mathbf{b}-\mathbf{g})$ The corresponding STM images at $V_{s}=-1,000 \mathrm{mV}$ exemplify the atom transfer ability using gate control for $(\mathbf{b}, \mathbf{c}) \mathrm{Au},(\mathbf{d}, \mathbf{e}) \mathrm{Ag}$ and $(\mathbf{f}, \mathbf{g}) \mathrm{Pb}$. The tunnelling current set points are (b,c) 30, (d,e) 50 and (f,g) 40 pA. The white dashed triangles mark the initial $(\mathbf{b}, \mathbf{d}$ and $\mathbf{f})$ and final $(\mathbf{c}, \mathbf{e}$ and $\mathbf{g})$ locations of adsorbates transferred by gate control. (h,i) AFM images showing $\mathrm{Sn}$ atom transfer by atom gating with AFM tip at $V_{\mathrm{s}}=0 \mathrm{~V}$. The acquisition parameters were $f_{0}=153,972.5 \mathrm{~Hz}, k=25.2 \mathrm{Nm}^{-1}, A=198 \AA$ and $\Delta f=-2.1 \mathrm{~Hz}$. 
a

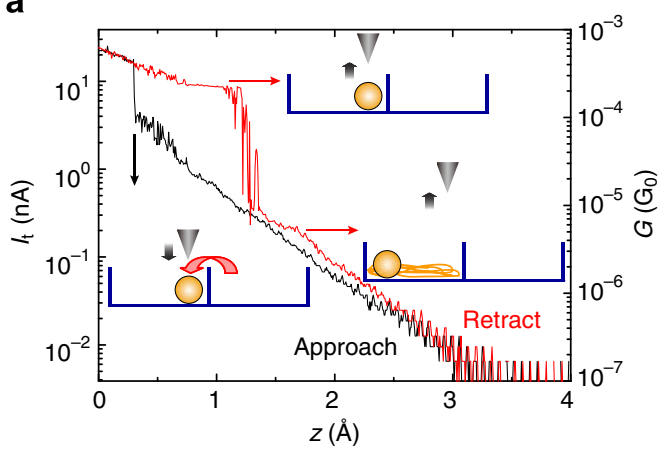

b

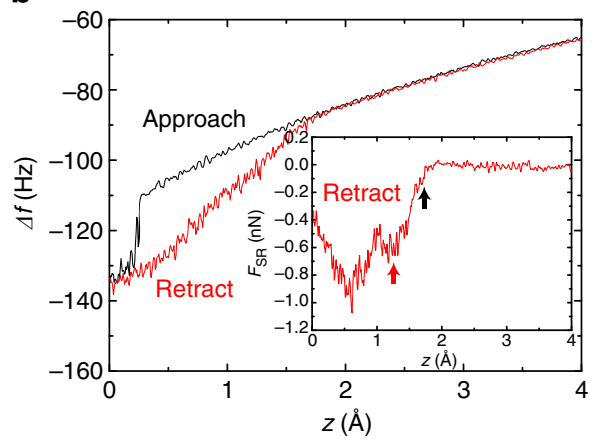

Figure 2 | STM and AFM observables during atom gating. (a) Typical $l_{t}(z)$ curves acquired with static STM at $V_{s}=-500 \mathrm{mV}$, revealing the evolution of the current (left axis) and conductance (right axis) during the Au atom gating process with the STM tip as a function of the tip-sample relative displacement. Here $G_{0}=2 e^{2} / h=(12,906 \Omega)^{-1}$. The spectroscopic measurements were performed over the site near the boundary of NSs, but slightly shifted away from the NS occupied by a Au atom. The inset schematics in a show the Au atom dynamics during the approach and retraction cycle of the tip (Au atom jump, Au atom trap and release). (b) The variation of the $\Delta f$ signal as a function of the tip-sample distance during approach and retraction acquired with AFM. The inset in $\mathbf{b}$ shows the short-range force extracted from the $\Delta f(z)$ curve, revealing the characteristic forces attributed to the trapping of a Au atom (black arrow) and junction formation by the chemical bond between the tip and the Au atom (red arrow). To extract the short-range force, the long-range background part, which is estimated from the approach $\Delta f(z)$ curve on the bare Si surface, was subtracted from the retraction $\Delta f(z)$ curve. It was then converted to the short-range force ${ }^{37}$. We define the origin of $z$ as the closest distance in data acquisition (no physical meaning). The acquisition parameters were $f_{0}=144,412.4 \mathrm{~Hz}, k=19.8 \mathrm{~N} \mathrm{~m}^{-1}, A=114 \AA$ and $V_{\mathrm{s}}=-500 \mathrm{mV}$.

distance is gradually decreased. In each gating attempt, this process is repeated until the diffusing atom is successfully transferred into the desired NS.

The scanning tunnelling microscopy (STM) images in Fig. 1b,c exemplify the gating process for a Au atom diffusing in a NS (marked by a white dashed triangle). Figure $1 \mathrm{~b}$ displays three Au atoms. Two of them are located in the faulted HUCs and one in the unfaulted HUC. Three HUCs with Au atoms appear brighter than the bare HUCs. This is attributable to the topographic as well as the electronic structure modifications induced by $\mathrm{Au}$ atoms that are diffusing much faster than the scanning speed of the SPM tip, similar to what was observed in a previous study ${ }^{21}$. Atom gating performed over the position marked by the cross in Fig. $1 \mathrm{~b}$ results in a transfer of a $\mathrm{Au}$ atom to an adjacent NS without any surface or tip structural modifications (see Fig. 1c). We achieved such atom transfers by atom gating for other elements, such as $\mathrm{Ag}, \mathrm{Pb}, \mathrm{Sn}$ and $\mathrm{Si}$ atoms (see Fig. 1d,e for $\mathrm{Ag}$ and Fig. 1f,g for Pb; also see Supplementary Fig. 1, Supplementary Note 2, Supplementary Movies 1 and 2). Although we have used only five different elements to demonstrate the gating process on the $\mathrm{Si}(111)-(7 \times 7)$ surface at $\mathrm{RT}$, this method can be applied, in principle, to other groups of elements. The main requirement that is needed for a successful gating process is the confinement of the adsorbates within a HUC without totally suppressing their mobility inside the HUCs.

Figure 1h,i shows AFM images demonstrating the ability to position a single $\mathrm{Sn}$ atom using atom gating without a tunnelling current $\left(I_{\mathrm{t}}\right)$ under $0 \mathrm{~V}$ bias. The $\mathrm{Sn}$ atom can be successfully relocated into the predetermined NS using an AFM tip. This is a strong evidence that the gating operation is not caused by $I_{\mathrm{t}}$.

Distance spectroscopy during atom gating. The dynamical evolution of the atomic process involved in atom gating can be obtained from the distance dependence of $I_{\mathrm{t}}\left(I_{\mathrm{t}}(z)\right)$. Figure $2 \mathrm{a}$ illustrates the representative semi-logarithmic $I_{\mathrm{t}}(z)$ curves recorded during the gating of Au by STM, which is acquired over the position marked by a cross in Fig. $1 \mathrm{~b}$. The $I_{\mathrm{t}}(z)$ curve follows the characteristic exponential increase with distance as the tip approaches above the bare Si surface (black curve). The most striking feature in Fig. $2 \mathrm{a}$ is the discontinuous jump observed at $z=0.3 \AA$. We ascribed the observed jump to the $\mathrm{Au}$ atom hopping from the initial NS to the adjacent empty NS below the tip; this can be explained as follows. Before the gate-opening process, the diffusing $\mathrm{Au}$ atom frequently visits adsorption sites close to the boundary between two NSs. When the gate is opened in response to the tip interaction at close proximity to the surface, the $\mathrm{Au}$ atom in the instant of closest approach to the boundary can hop to the adjacent NS. The Au atom is then locally trapped under the STM tip by a chemical bonding force. Thereby an atomic junction of the tip-Au-Si surface is formed. The formation of this atomic junction gives rise to a sudden increase in $I_{\mathrm{t}}$, as encountered in our experiments. As the tip-surface separation further decreases $(z<0.3 \AA), I_{\mathrm{t}}$ increases with a smaller slope, which signifies that the tunnelling barrier collapses and ballistic current starts to flow through the junction.

During the tip retraction (red curve), $I_{\mathrm{t}}(z)$ follows the approach curve in the range of $z<0.3 \AA$, and continues to decrease with a smaller slope beyond the distance where the $\mathrm{Au}$ atom hopped. A plateau region is observed around $z=1 \AA$, where the Au atom is still trapped and the atomic junction is preserved. This atomic junction is stabilized by the chemical bonding force, which is evident in the relatively low noise level in the plateau region (further discussion can be found in Supplementary Note 3). We obtained $G=2.2 \times 10^{-4} G_{0}$ as the quantized conductance through a single $\mathrm{Au}$ atomic junction, which is much smaller than the one obtained in pure gold atomic junctions where the conductance is close to $1 G_{0}$ (ref. 22). The observed small conductance was attributed to the energy level mismatch between the $\mathrm{Au}$ and $\mathrm{Si}$ surfaces (further discussion can be found in Supplementary Note 4).

As the tip is retracted further from the plateau region, the $I_{\mathrm{t}}(z)$ curve fluctuated around $z=1.2 \AA$. The fluctuations suggest the presence of a stochastic trap and release of the Au atom in the junction by the tip. Beyond this transition region, the chemical bond arising between the $\mathrm{Au}$ atom and the tip is completely broken, and the Au atom thermally diffuses within the NS all of the time. Consequently, the $I_{\mathrm{t}}$ value drops by one order of magnitude. Hereafter, $I_{\mathrm{t}}$ decreases as the tip-to-surface distance increases following the basic tunnelling law. The characteristic features observed in the $I_{\mathrm{t}}(z)$ curves are remarkably identical for different elements used for cluster assembling, thus pointing towards a common mechanism for atom gating. 


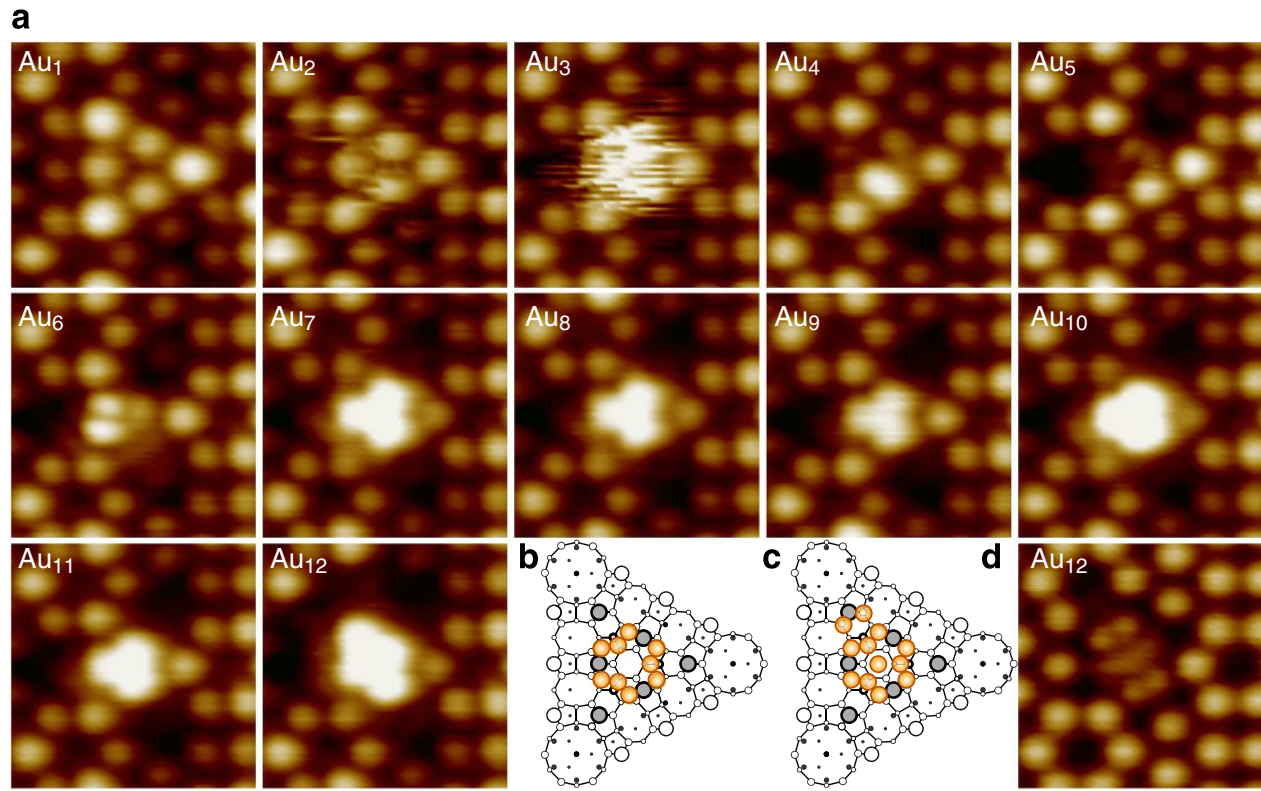

Figure 3 | Assembly of $\mathbf{A u}$ atom clusters by the successive atom gating. (a) STM filled-state images of typical $A u_{1}-A u_{12}$ clusters assembled by gate control (acquired at $V_{s}=-500 \mathrm{mV}$ and $I_{t}=30 \mathrm{pA}$ ). (b) Schematic representation of the structure model for Aug effectuated by Ghose et al. ${ }^{27}$ In this model, six Au atoms are located inside the basin (close to the $\mathrm{H} 3$ site) and the other three reside over the symmetry axes by bonding to the other $\mathrm{Au}$ atoms. Au atoms do not participate in bond formation with corner adatoms. (c) Schematic representation of a possible structure model for $\mathrm{Au}_{12}$. (d) STM empty-state image of $\mathrm{Au}_{12}$ cluster at $V_{\mathrm{s}}=1,000 \mathrm{mV}$ and $I_{\mathrm{t}}=40 \mathrm{pA}$.

To further elucidate the triggering mechanism of the gating and to quantify the force required to trap the diffusing Au atom under the tip, we performed force spectroscopic measurements using AFM/STM. We simultaneously recorded frequency shift $(\Delta f)$, current and energy dissipation during a gating operation (Fig. 2b and Supplementary Fig. 2). When the oscillating tip approaches the surface for atom gating, all recorded signals jump at around $z=0.3 \AA$, which is the signature of a $A u$ atom hopping from a neighbouring NS. It should be pointed out that once the Au atom is transferred to the NS below the tip, the tip traps and releases the $\mathrm{Au}$ atom in each tip oscillation cycle owing to the large cantilever oscillation amplitude used in our experiment (see Supplementary Note 5 and Supplementary Movie 3).

We determined the threshold force required to trap a single diffusing $\mathrm{Au}$ atom below the tip as $F_{\mathrm{SR}}=-0.1 \mathrm{nN}$. This was obtained from the short-range force during tip retraction at the distance above which the trapped $\mathrm{Au}$ atom diffuses freely within the NS without any further trapping by the tip (see the black arrow in the inset of Fig. 2b, Supplementary Fig. 2 and Supplementary Note 5). Moreover, the interaction force stabilizing the atomic junction between the tip and the Au atom can be estimated to be $F_{\mathrm{SR}}=-0.6 \mathrm{nN}$ (marked by the red arrow), which is in the class of a chemical bonding force ${ }^{23}$. At closer distances, the second force minimum appears in $F_{\mathrm{SR}}(z)$ curve, which can be attributed to relaxations of the tip apex (see Supplementary Fig. 2 and Supplementary Note 5). From the measured force magnitude and the ability to transfer single adsorbates without tunnelling current (see Fig. 1h,i), we conclude that the gating mechanism is a purely mechanical effect. Here the term 'mechanical effect' indicates that the tunnelling current and the electric field do not play any role in the atom gating process. The formation of the chemical bond between the outermost apex atom and the adsorbate atom on the surface weakens the adsorbate bonds with the underlying $\mathrm{Si}$ atoms $\mathrm{s}^{16}$, leading to a local reduction of the energy barrier so that thermal energy enables atom hopping across the gate.
Assembly of clusters composed of a defined number of atoms. We can fabricate complex atom clusters by the gating technique as described above. Movies showing the assembly of these clusters are available in Supplementary Movies 4-9. An example, the assembly of $\mathrm{Au}_{N}$ clusters $(N=1-12)$ with most stable forms, is demonstrated in Fig. 3a. These clusters were assembled by bringing $\mathrm{N} \mathrm{Au}$ atoms into the predetermined NS one-by-one by successive gate operations (see Supplementary Note 6). Atom-byatom assembly allows us to study the dependence of the cluster stability on the size of clusters or the number of atoms that compose them. While $\mathrm{Au}_{1}-\mathrm{Au}_{3}$ shows quick thermal diffusion, $\mathrm{Au}_{4}$ and $\mathrm{Au}_{7}-\mathrm{Au}_{12}$ remain stable structures and no changes were observed in their appearance within several hours. On the other hand, $\mathrm{Au}_{5}$ and $\mathrm{Au}_{6}$ are relatively stable within the scanning time scale, but in several minutes, conformational changes in the structures of the clusters into the equivalent forms take place by following the mirror and threefold symmetry of the $\mathrm{Si}(111)-(7 \times 7)$ surface. This type of transformation takes place by collective diffusion of the cluster atoms.

$\mathrm{Au}_{7}-\mathrm{Au}_{11}$ atom clusters look identical to each other, with threefold symmetry in the filled-state STM images, and they resemble the self-assembled Au clusters previously observed in refs 24,25 . In that study ${ }^{25}$, the structure model was determined based on the first-principle calculations and the number of $\mathrm{Au}$ atoms in the clusters was estimated as six, which is not consistent with our results. Previous investigations showed that metallic elements such as $\mathrm{Au}$ and $\mathrm{Ag}$ interact with multiple $\mathrm{Si}$ atoms to saturate the maximum number of dangling bonds by adsorbing at high coordination $\operatorname{sites}^{26}$. A favourable structure model for $\mathrm{Au}_{9}$ cluster was previously proposed (also depicted in Fig. 3b) on the basis of X-ray diffraction experiments ${ }^{27}$. The lack of their participation in chemical bonding with corner adatoms and their three-lobe appearance in our STM images suggest that this structure model can serve as a possible basis for structure models for $\mathrm{Au}_{7}-\mathrm{Au}_{11}$. In contrast, the apparent threefold symmetry is broken for $\mathrm{Au}_{12}$ since one of the corner adatoms starts to participate in bond formation. The $\mathrm{Au}_{12}$ structure still has a 
a

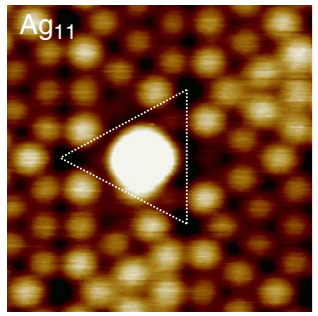

e

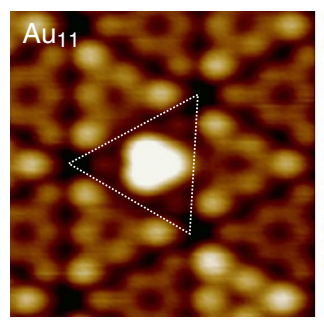

i

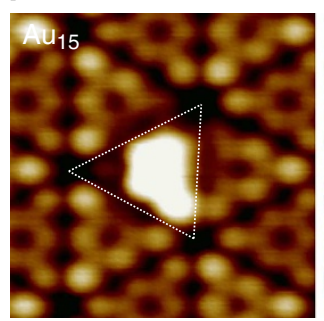

I

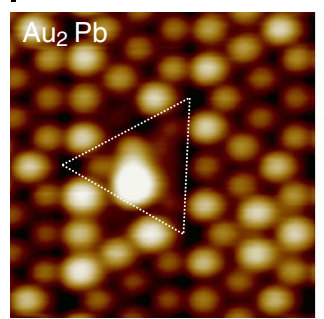

b

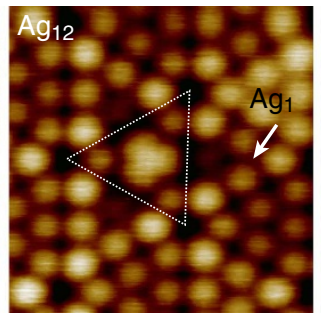

C

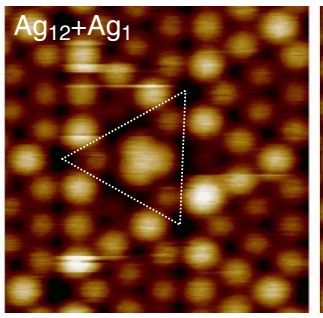

d

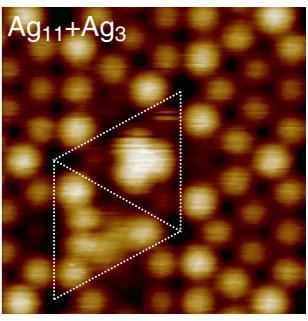

h

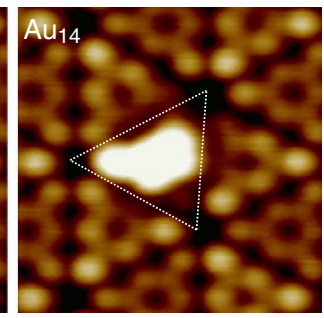

g

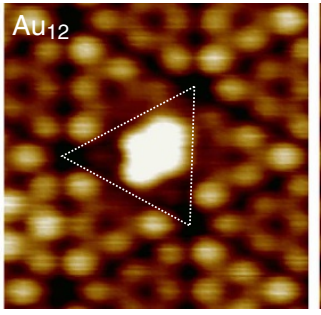

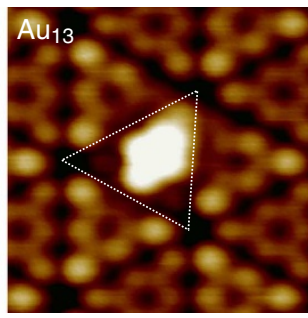

k
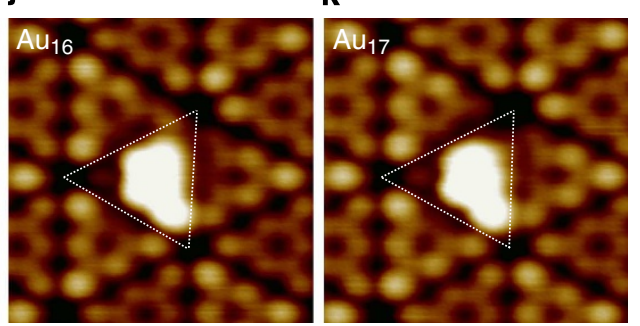

m

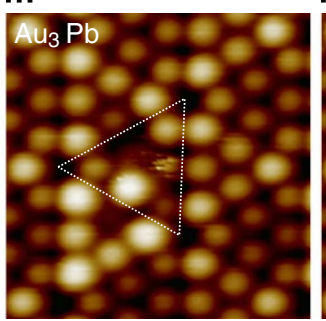

n

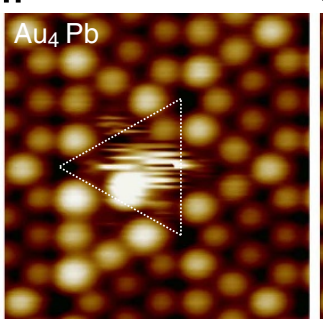

o

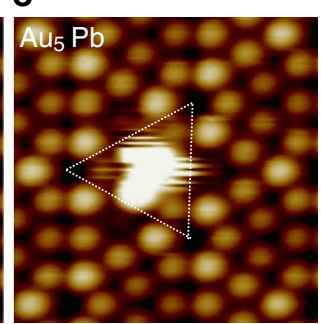

Figure 4 | The dependence of the cluster stability on composition. (a-d) Series of STM images showing the influence of Ag atom transfer into a NS nearby to the existing $\mathrm{Ag}$ cluster formed at the unfaulted $\mathrm{HUC}$ of $\mathrm{Si}(111)-(7 \times 7)$. (a) $\mathrm{Ag}_{11}$; (b) transfer of one $\mathrm{Ag}$ atom into an adjacent NS leads to the formation of $\mathrm{Ag}_{12}$; (c) transfer of an additional $\mathrm{Ag}$ atom into a neighbouring NS occupied by $\mathrm{Ag}_{12}$ does not allow $\mathrm{Ag}_{13}$ cluster formation; (d) addition of two $\mathrm{Ag}$ atoms into the neighbouring NS occupied by $A g_{12}$ creates two cluster arrays of $A g_{11}$ and $A g_{3}$. (e-k) STM images showing the $A u_{11}-A u_{17}$ clusters assembled at the unfaulted HUC. (I-o) STM images of multi-element atom clusters showing the influence of a single $\mathrm{Pb}$ atom on the stability of $\mathrm{Au}_{2}-\mathrm{Au}_{5}$ clusters assembled by atom gating control. The acquisition parameters were (a-d) $V_{s}=-1,000 \mathrm{mV}$ and $I_{\mathrm{t}}=50 \mathrm{pA} ;(\mathbf{e}-\mathbf{k}) V_{\mathrm{s}}=-1,000 \mathrm{mV}$ and $I_{\mathrm{t}}=30 \mathrm{pA}$; (I-o) $V_{\mathrm{s}}=-1,000 \mathrm{mV}$ and $I_{\mathrm{t}}=30 \mathrm{pA}$.

mirror symmetry in both filled-and empty-state STM images (see Fig. 3d). On the basis of this information and the proposed model for $\mathrm{Au}_{9}$ cluster $^{27}$, we propose a structure model for $\mathrm{Au}_{12}$ cluster. The two Au atoms are placed near a corner Si adatom site, and are located symmetrically with respect to the mirror plane. The additional one $\mathrm{Au}$ atom is placed at the centre of the HUC. The resulting structure model shown in Fig. $3 \mathrm{c}$ fulfils a mirror symmetry as observed by the STM.

The precise control of single $\mathrm{Au}$ atoms as demonstrated here enables us to determine the exact number of atoms forming each cluster, thus providing accurate information for future calculations to construct possible structure models. Although we did not determine the capacity of a HUC that can accommodate maximum number of $\mathrm{Au}$ atoms, we could assemble Au clusters containing up to $17 \mathrm{Au}$ atoms (see Fig. 4e-k).
The cluster structures and their stabilities are strongly dependent on the elements that constitute the clusters. Figure $4 \mathrm{a}-\mathrm{d}$ and Fig. 4e-k compare the stability of distinct types of clusters formed by the individual $\mathrm{Ag}$ and $\mathrm{Au}$ atoms, respectively. The assembly sequences of these clusters can be found in Supplementary Notes 6 and 7. $\mathrm{Ag}_{12}$ formed by atom gating is shown in Fig. $4 \mathrm{~b}$. A threefold symmetry in the structure of the $\mathrm{Ag}_{12}$ cluster is observed, which is clearly different from $\mathrm{Au}_{12}$ (Fig. 4f), although both $\mathrm{Ag}$ and $\mathrm{Au}$ belong to the same chemical group. More remarkably, $\mathrm{Ag}_{N}$ with $N \geq 13$ never formed at an unfaulted $\mathrm{HUC}$, whereas $\mathrm{Au}_{13}-\mathrm{Au}_{17}$ did form (see Fig. $4 \mathrm{~g}-\mathrm{k}$ ). When a diffusing $\mathrm{Ag}$ atom is transferred into a NS adjacent to $\mathrm{Ag}_{12}$ as the arrow in Fig. 4b shows, it hops out quickly and diffuses among the three nearest neighbouring NSs surrounding $\mathrm{Ag}_{12}$ (see Fig. 4c). This suggests that the barrier 
associated with the single $\mathrm{Ag}$ atom diffusion to the nearest neighbouring NSs can be lowered by the presence of the $\mathrm{Ag}_{12}$ cluster even without the tip's close proximity effect (see Supplementary Fig. 3, and Supplementary Notes 7 and 8). Although the route for these diffusions had to pass through the NS that held the $\mathrm{Ag}_{12}$ cluster, $\mathrm{Ag}_{13}$ never formed within the observable time scale. This is an indication that 12 is the maximum number of $\mathrm{Ag}$ atoms available for each NS of an unfaulted HUC.

It is interesting to note that, when two Ag atoms are brought into a NS adjacent to $\mathrm{Ag}_{12}, \mathrm{Ag}_{3}$ is formed instead of $\mathrm{Ag}_{2}$, as shown in Fig. $4 \mathrm{~d}$. The presence of the $\mathrm{Ag}_{2}$ cluster in the nearby cell leads to a dissociation of one $\mathrm{Ag}$ atom from the $\mathrm{Ag}_{12}$, thereby producing two cluster arrays of $\mathrm{Ag}_{11}$ and $\mathrm{Ag}_{3}$. The resulting $\mathrm{Ag}_{3}$ diffuses in a concerted way among the three NSs surrounding the newly formed $\mathrm{Ag}_{11}$ cluster. The appearance of the $\mathrm{Ag}_{11}$ cluster accompanied by the formation of $\mathrm{Ag}_{3}$ looks different from the isolated $\mathrm{Ag}_{11}$ (compare Fig. $4 \mathrm{a}$ and Fig. 4d; also see Supplementary Figs 3 and 4, and Supplementary Notes 7 and 9).

A preferential growth in the faulted HUC has been observed for the $\mathrm{Au}$ and $\mathrm{Ag}$ clusters formed by thermal annealing process in previous STM investigations ${ }^{9,25}$. On the contrary, with our gating method the clusters can be assembled equally on both the faulted and unfaulted HUCs, since the adsorbates are forcibly collected into a predetermined HUC.

The fabrication method provided by the gating process can also be used to assemble multi-element clusters. The additional degree of freedom inherent in bimetallic clusters offers the opportunity to tune their structural and chemical properties by varying the cluster composition. The composition in the cluster can be designed and controlled atom-by-atom by identifying the atomic species adsorbed sequentially on surfaces from the SPM images.

The bimetallic clusters involving metal atoms of two different species were assembled (see Supplementary Fig. 5 and Supplementary Note 10). An exemplary cluster of $\mathrm{Au}_{N} \mathrm{~Pb}$ (with $N=2-5$ ) is displayed in Fig. 4l-o. The characteristic appearance of these mixed clusters departs considerably from their pure counterparts (see Fig. 2 and Supplementary Notes 6 and 11). The presence of the single $\mathrm{Pb}$ atom in $\mathrm{Au}_{2}$ can stabilize the $\mathrm{Au}_{2} \mathrm{~Pb}$ cluster, although $\mathrm{Au}_{2}$ and $\mathrm{Au}_{3}$ are mobile at RT. On the contrary, the $\mathrm{Au}_{4} \mathrm{~Pb}$ and $\mathrm{Au}_{5} \mathrm{~Pb}$ clusters exhibit two distinct features separated by partially stable round and fuzzy features in the STM images, whereas $\mathrm{Au}_{4}-\mathrm{Au}_{6}$ are relatively stable (see Fig. 3a). This result implies that a single $\mathrm{Pb}$ atom can stabilize a maximum of only two Au atoms in the NS. Accommodation of an additional number of $\mathrm{Au}$ atoms into this NS does not contribute to the clustering with $\mathrm{Au}_{2} \mathrm{~Pb}$, leaving the added $\mathrm{Au}$ atoms free to move. Thus, the $\mathrm{Au}_{2} \mathrm{~Pb}$ cluster can be classified as a magic cluster due to its preference for staying intact without interaction with other $\mathrm{Au}$ atoms.

\section{Discussion}

These exemplary results clearly indicate that the stability of the clusters depends critically on the atomic species involved in the cluster structures. Valency of each component in a cluster dominates the bonding network and therefore determine the stability of the cluster. This fabrication technique can potentially be applied to the investigation of synergistic effects in bimetallic clusters $^{28}$. It might be also possible to tune the reactivities of bimetallic clusters by varying the nature of the constituent atoms using the atom gating method, allowing the study of the reactivities of subnanoscale catalysis.

We have demonstrated a new approach to the formation of various atom clusters with pre-designed composition at RT. The mechanism underlying this method was identified as a pure mechanical effect. The tip induces a reduction of the activation energy barrier between the adjacent NSs, allowing the diffusing atoms to jump into the desired NS, accompanied by atom trapping below the tip. This method provides access to investigate the dependence of a cluster's stability on its size and composition. This technique, when combined with simultaneous AFM/STM characterization ability of submolecular level imaging ${ }^{29}$ and of chemical identification ${ }^{30}$, may allow us to experimentally determine the structures of clusters. Atom gating is a highly controllable technique that can be applied to various adsorbates and surfaces possessing periodic NS arrays, such as graphene moiré pattern ${ }^{31}$, hexagonal boron nitride (h-BN) nanomesh ${ }^{32}$ and alumina on $\mathrm{Ni}_{3} \mathrm{Al}(111)$ templates ${ }^{33}$. The cluster arrays formed on these templates are expected to have potential in both fundamental aspects and technological applications. These results serve as the basis for further investigations of the interplay between the sizes of supported clusters and their magnetic and catalytic properties.

\section{Methods}

AFM/STM set-up and sample preparation. The experiments were carried out with custom-built ultra-high-vacuum AFM/STM systems operated at RT ${ }^{15,18}$, equipped with versatile scan controllers ${ }^{34}$. Commercial Pt-Ir-coated Si cantilevers (NCLPt, NanoWorld) were used for static STM measurements (that is, without tip oscillation) and simultaneous AFM/STM measurements (that is, dynamic mode). For the particular case of AFM measurements, Si cantilevers without a metal coating were also used. Before use, the tip apex in both types of cantilevers was carefully cleaned by Ar ion sputtering. Cantilever deflection sensors are based on optical interferometers. For AFM measurements, we applied the frequency modulation detection method ${ }^{35}$, by which we detected $\Delta f$ of cantilevers oscillating at a constant amplitude. Bias voltage $\left(V_{s}\right)$ was applied to the sample with respect to the tip being held at virtual ground potential. The $I_{\mathrm{t}}$ signal was measured from the tip. The experimental set-up is described in detail elsewhere ${ }^{36}$. Acquisition parameters are summarized in Supplementary Table. The $\operatorname{Si}(111)-(7 \times 7)$ surface was prepared by a standard method of flashing and annealing. Various elements $(\mathrm{Si}, \mathrm{Sn}, \mathrm{Pb}, \mathrm{Ag}$ and $\mathrm{Au}$ ) were deposited onto the $\mathrm{Si}(111)-(7 \times 7)$ surface by using a Joule heating evaporator in an ultra-high vacuum while the sample temperature was kept at RT (no further annealing process).

Details of atom gating procedure. The close proximity of the tip to the surface clearly affects atom transferring across the boundary. The success rate increases drastically with decreasing the tip-surface distance for atom gating process. A successful atom transferring by means of atom gating process takes place when the tip is approached towards the surface by about $3 \AA$ from STM feedback position. We have empirically found that the location indicated by a cross in Fig. 1a is the most efficient site for transferring of the adsorbates presented in this study. On the other hand, the speed of vertical tip movement during the approach and retraction can also contribute to the probability of atom hopping between two NSs. The residence time of tip interaction with the target atom at the distance of closest approach to the surface increases by reducing the speed of vertical tip approach/ retraction. Consequently, the rate of the atom hopping probability increases.

\section{References}

1. Klein, D. L., Roth, R., Lim, A. K. L., Alivisatos, A. P. \& McEuen, P. L. A single-electron transistor made from a cadmium selenide nanocrystal. Nature 389, 699-701 (1997).

2. Ray, V. et al. CMOS-compatible fabrication of room temperature single-electron devices. Nat. Nanotechnol. 3, 603-608 (2008).

3. Haruta, M., Yamada, N., Kobayashi, T. \& Iijima, S. Gold catalysts prepared by coprecipitation for low-temperature oxidation of hydrogen and of carbon monoxide. J. Catal. 115, 301-309 (1989).

4. Haruta, M. Size- and support-dependency in the catalysis of gold. Catal. Today 36, 153-166 (1997).

5. Valden, M., Lai, X. \& Goodman, D. W. Onset of catalytic activity of gold clusters on titania with the appearance of nonmetallic properties. Science 281, 1647-1650 (1998).

6. Ladd, T. D. et al. Quantum computers. Nature 464, 45-53 (2010).

7. Brune, H., Giovannini, M., Bromann, K. \& Kern, K. Self-organized growth of nanostructure arrays on strain-relief patterns. Nature 394, 451-453 (1998).

8. Li, J. L. et al. Spontaneous assembly of perfectly ordered identical-size nanocluster arrays. Phys. Rev. Lett. 88, 066101 (2002).

9. Li, S. C., Jia, J. F., Dou, R. F. \& Xue, Q. K. Borderline magic clustering: The fabrication of tetravalent $\mathrm{Pb}$ cluster arrays on $\mathrm{Si}(111)-(7 \times 7)$ surfaces. Phys. Rev. Lett. 93, 116103 (2004). 
10. Eigler, D. M. \& Schweizer, E. K. Positioning single atoms with a scanning tunnelling microscope. Nature (London) 344, 524-526 (1990).

11. Nilius, N., Wallis, T. M. \& Ho, W. Development of one-dimensional band structure in artificial gold chains. Science 297, 1853-1856 (2002)

12. Stroscio, J. A., Tavazza, F., Crain, J. N., Celotta, R. J. \& Chaka, A. M. Electronically induced atom motion in engineered CoCun nanostructures. Science 313, 948 (2006).

13. Loth, S., Baumann, S., Lutz, C. P., Eigler, D. M. \& Heinrich, A. J. Bistability in atomic-scale antiferromagnets. Science 335, 196-199 (2012).

14. Khajetoorians, A. A. et al. Current-driven spin dynamics of artificially constructed quantum magnets. Science 339, 55-59 (2013).

15. Sugimoto, Y. et al. Atom inlays performed at room temperature using atomic force microscopy. Nat. Mater. 4, 156-159 (2005).

16. Sugimoto, Y. et al. Mechanism for room-temperature single-atom lateral manipulations on semiconductors using dynamic force microscopy. Phys. Rev. Lett. 98, 106104 (2007).

17. Ternes, M., Lutz, C. P., Hirjibehedin, C. F., Giessibl, F. J. \& Heinrich, A. J. The force needed to move an atom on a surface. Science 319, 1066-1069 (2008).

18. Sugimoto, Y. et al. Complex patterning by vertical interchange atom manipulation using atomic force microscopy. Science 322, 413-417 (2008).

19. Ming, F. et al. Assembling and disassembling Ag clusters on $\operatorname{Si}(111)-(7 \times 7)$ by vertical atomic manipulation. ACS Nano 5, 7608-7616 (2011).

20. Gomez-Rodriguez, J. M., Saenz, J. J. \& Baro, A. M. Real-time observation of the dynamics of single $\mathrm{Pb}$ atoms on $\mathrm{Si}(111)-(7 \times 7)$ by scanning tunneling microscopy. Phys. Rev. Lett. 76, 799-802 (1996).

21. Wang, K., Zhang, C., Loy, M. M. T. \& Xiao, X. Time-dependent tunneling spectroscopy for studying surface diffusion confined in nanostructures. Phys. Rev. Lett. 94, 036103 (2005).

22. Ohnishi, H., Kondo, Y. \& Takayanagi, K. Quantized conductance through individual rows of suspended gold atoms. Nature 395, 780-783 (1998).

23. Yurtsever, A. et al. Force mapping on a partially H-covered $\operatorname{Si}(111)-(7 \times 7)$ surface: Influence of tip and surface reactivity. Phys. Rev. B 87, 155403 (2013).

24. Chizhov, I., Lee, G. \& Willis, R. F. Initial stages of Au adsorption on the Si(111)- $(7 \times 7)$ surface studied by scanning tunneling microscopy. Phys. Rev. B 56, 12316-12320 (1997).

25. Wu, Y., Zhou, Y., Zhou, C., Zhan, H. \& Kang, J. Atomic structure and formation mechanism of identically sized Au clusters grown on $\operatorname{Si}(111)-(7 \times 7)$ surface. J. Chem. Phys. 133, 124706 (2010).

26. Zhang, C. et al. Experimental and theoretical investigation of single $\mathrm{Cu}, \mathrm{Ag}$, and $\mathrm{Au}$ atoms adsorbed on Si(111)-(7 × 7). Phys. Rev. Lett. 94, 176104 (2005).

27. Ghose, S. K., Bennett, P. A. \& Robinson, I. K. Clustering of Au on the faulted half of the Si(111)-7 × 7 unit cell. Phys. Rev. B 71, 073407 (2005).

28. Zhang, H., Watanabe, T., Okumura, M., Haruta, M. \& Toshima, N. Catalytically highly active top gold atom on palladium nanocluster. Nat. Mater. 11, 49-52 (2011).
29. Gross, L., Mohn, F., Moll, N., Liljeroth, P. \& Meyer, G. The chemical structure of a molecule resolved by atomic force microscopy. Science 325, 1110-1114 (2009).

30. Sugimoto, Y. et al. Chemical identification of individual surface atoms by atomic force microscopy. Nature (London) 446, 64-67 (2007).

31. Franz, D. et al. Atomic structure and crystalline order of graphene-supported Ir nanoparticle lattices. Phys. Rev. Lett. 110, 065503 (2013).

32. Zhang, J. et al. Ordered layers of Co clusters on BN template layers. Phys. Rev. B 78, 165430 (2008).

33. Buchsbaum, A., Santis, M. D., Tolentino, H. C. N., Schmid, M. \& Varga, P. Highly ordered $\mathrm{Pd}, \mathrm{Fe}$, and Co clusters on alumina on $\mathrm{Ni}_{3} \mathrm{Al}(111)$. Phys. Rev. $B$ 81, 115420 (2010).

34. Horcas, I. et al. Wsxm: A software for scanning probe microscopy and a tool for nanotechnology. Rev. Sci. Instrum 78, 013705 (2007).

35. Albrecht, T. R., Grütter, P., Horne, D. \& Rugar, D. Frequency modulation detection using high-q cantilevers for enhanced force microscope sensitivity. J. Appl. Phys. 69, 668-673 (1991).

36. Sugimoto, Y. et al. Simultaneous AFM and STM measurements on the Si(111)-(7×7) surface. Phys. Rev. B 81, 245322 (2010).

37. Sader, J. E. \& Jarvis, S. P. Accurate formulas for interaction force and energy in frequency modulation force spectroscopy. Appl. Phys. Lett. 84, 1801-1803 (2004).

\section{Acknowledgements}

This work was supported by a Grant-in-Aid for Scientific Research (18860046, 22221006, 24360016, 25106002, 26110516, 26600015 and 26600099) from the Ministry of Education, Culture, Sports, Science and Technology of Japan (MEXT), Funding Program for Next Generation World-Leading Researchers.

\section{Author contributions}

Y.S. conceived and designed the experiments; Y.S. and N.H. performed the experiments; Y.S., A.Y., M.A, and S.M. analysed the data; Y.S. and A.Y. co-wrote the paper. All authors discussed the results and commented on the manuscript.

\section{Additional information}

Supplementary Information accompanies this paper at http://www.nature.com/ naturecommunications

Competing financial interests: The authors declare no competing financial interests.

Reprints and permission information is available online at http://www.nature.com/ reprintsandpermissions/

How to cite this article: Sugimoto, Y. et al. Mechanical gate control for atom-by-atom cluster assembly with scanning probe microscopy. Nat. Commun. 5:4360 doi: 10.1038/ ncomms5360 (2014). 